\title{
Essai d'une farine de sevrage dans la réhabilitation nutritionnelle de la malnutrition aigüe en milieu communautaire, au Niger
}

\author{
Maman Mahaman Salissou', Bako Aminatou Maazou² et Sabo Haoua Seini ${ }^{3}$ \\ 1: Département de chimie-Biochimie, Nutrition Humaine, Faculté des sciences et Techniques / Université Abdou \\ Moumouni de Niamey au Niger Email : saliss7@yahoo.fr, Tel : (+227) 96513620 ou (+227) 81002131. \\ 2: Département des Sciences Biologiques Appliquées, Faculté des Sciences de la Santé / Université Abdou \\ Moumouni de Niamey au Niger Email : bako.aminatou@gmail.com Tel : (+227) 96100356 \\ 3 : Département de chimie-Biochimie, Faculté des sciences et Techniques / Université Abdou Moumouni de Niamey \\ au Niger.
}

Original submitted in on $11^{\text {th }}$ March 2020. Published online at www.m.elewa.org/journals/ on $31^{\text {st }} \mathrm{July} 2020$ https://doi.org/10.35759/JABs.151.6

\section{RÉSUMÉ}

Objectif : l'objectif vise à évaluer l'efficacité des bouillies à base de mil, tourteaux d'arachide et la poudre de moringa, chez les enfants âgés de 6 à 59 mois admis pour malnutrition au niveau du Centre de Santé Intégré de Dan Issa.

Méthodologie et Résultats : Cette étude prospective, s'est déroulée du 23 août au 04 octobre 2019. Elle a concerné 44 enfants de 06 à 59 mois, répartis en trois groupes. Le premier groupe de 20 enfants atteints de malnutrition aigüe modérée, recevant la bouillie ; le deuxième groupe constitué de 12 enfants atteints de malnutrition aigüe légère, reçoit la même bouillie et le troisième groupe constitué de 12 enfants atteints de malnutrition aigüe modérée, reçoit du CSB++. Les données ont été analysées avec le logiciel SPSS version 20. Ainsi, il ressort : pour le premier groupe, l'âge moyen est de 12,20 mois $\pm 4,336$. La moitié des enfants $(50 \%)$ a observé une amélioration de leur nutritionnel dès la première semaine de traitement avec un gain de poids moyen de 73,92g par jour $\pm 73,7$. Pour le deuxième groupe, l'âge moyen est de 28,92 mois $\pm 15,163$. On a constaté que $75 \%$ des enfants ont atteint le poids cible dès la première semaine de prise en charge, avec un gain de poids moyen de $85,11 \mathrm{~g} / \mathrm{j} \pm 47,22$. Pour le troisième groupe ayant reçu le CSB++, on a constaté que seuls 3 enfants sur $12(25 \%)$ ont observé une amélioration de leur état nutritionnel après 14 jours de traitement. Le gain de poids moyen était de $20,53 \mathrm{~g} / \mathrm{j} \pm 23,14$. Cependant, il faut noter que ce dernier groupe n'a reçu le CSB++ qu'une seule fois et le CSI était en rupture jusqu'à la fin de l'étude. En effet, l'action de la bouillie sur le gain pondéral a été remarquable. Tous les enfants ont acceptés la bouillie et qu'aucun signe d'intolérance n'a été noté.

Conclusion et application des résultats : la bouillie mis au point à partir du mil, tourteaux d'arachide et la poudre de moringa est une approche pour combattre ou prévenir à long terme la malnutrition chez les enfants de moins de cinq ans au Niger. Cependant, la bouillie seule ne constitue pas le traitement, mais doit être couplée avec des mesures sanitaires, d'hygiène et d'éducation nutritionnelle des mères.

Mots-clés : essai, farine, malnutrition, prévention, réhabilitation nutritionnelle, Niger. 


\section{ABSTRACT}

Objective: the objective aims to assess the effectiveness of millet-based porridge, peanut cake and moringa powder, in children aged 6 to 59 months admitted for malnutrition at the Dan Issa Integrated Health Center.

Methodology and Results: This prospective study took place from August 23 to October 04, 2019. It involved 44 children from 06 to 59 months, divided into three groups. The first group of 20 children with moderate acute malnutrition, receiving porridge; the second group of 12 children with mild acute malnutrition receives the same porridge and the third group of 12 children with moderate acute malnutrition receives CSB ++. Data were analyzed with SPSS software version 20. Thus, it emerges: for the first group, the average age is 12.20 months \pm 4.336 . Half the children (50\%) observed an improvement in their nutrition from the first week of treatment with an average weight gain of $73.92 \mathrm{~g}$ per day \pm 73.7 . For the second group, the average age is 28.92 months \pm 15.163 . It was found that $75 \%$ of children reached the target weight within the first week of treatment, with an average weight gain of $85.11 \mathrm{~g} / \mathrm{d} \pm 47.22$. For the third group who received CSB ++, it was found that only 3 out of 12 children $(25 \%)$ observed an improvement in their nutritional status after 14 days of treatment. The average weight gain was $20.53 \mathrm{~g} / \mathrm{d}$ \pm 23.14 . However, it should be noted that the latter group received CSB ++ only once and the CSI was out until the end of the study. Indeed, the action of porridge on weight gain has been remarkable. All the children accepted the porridge and no signs of intolerance were noted.

Conclusion and application of the results: the porridge developed from millet, peanut cake and moringa powder is an approach to combat or prevent long-term malnutrition in children under five in Niger. However, porridge alone is not the treatment, but should be combined with sanitary, hygienic and nutritional education measures for mothers.

Keywords: trial, flour, malnutrition, prevention, nutritional rehabilitation, Niger.

\section{INTRODUCTION}

La malnutrition et les carences en nutriments associées constituent l'un des plus grands problèmes récurrents de santé publique des zones pauvres du Niger (INS, 2019). Cette malnutrition est liée aux déficits alimentaires chroniques observés dans plusieurs zones vulnérables (OCHA, 2016). Une alimentation et une nutrition adéquates pendant la petite enfance sont des conditions essentielles pour assurer à un pays des ressources humaines en bonne santé, condition nécessaire à un développement durable (OMS, 2006). Selon le rapport d'enquête nationale $d u$ Niger réalisé en 2019 , la prévalence nationale de la malnutrition chronique est de $47,8 \%$ chez les enfants de moins de 5 ans. Celle de la malnutrition aigüe globale est de $15,0 \%$. Ces taux correspondent à une situation critique selon la classification de l'OMS. Les mauvaises conditions d'hygiène chez les jeunes enfants augmentent le risque de contracter des maladies, en particulier les maladies diarrhéiques qui affectent, à leur tour l'état nutritionnel de l'enfant. Une alimentation inadéquate et un environnement sanitaire inadéquat sont le reflet de la précarité des conditions socio-économiques et nutritionnelles (OMS, 2006). Les conséquences immédiates d'une nutrition insuffisante au cours de ces années de développement comprennent une morbidité et une mortalité augmentées, ainsi qu'un retard du développement psychomoteur (OMS, UNICEF, 2009). De nombreuses stratégies ont été mises en œuvre par le Ministère de la Santé Publique et ses partenaires en vue d'améliorer l'état nutritionnel des enfants. Cependant, la prévalence de la malnutrition reste toujours élevée. Dans cette étude, les auteurs ont proposé une farine, afin d'en tester l'efficacité et la tolérance dans le traitement diététique de la malnutrition aigüe de l'enfant.

Présentation du produit : La farine est un produit à base de céréales (mil à $60 \%$ ), de légumineuses (tourteaux d'arachide à 20\%), de la poudre de moringa oleifera $(10 \%)$ et sucre $(10 \%)$. Elle a été fabriquée grâce à la collaboration du projet 
MISOLA, I'Unité de Production de Tessaoua (UPT). La farine est conditionnée en sachet de 200 gramme avec une teneur protidique de 31,66 gramme et une valeur énergétique de $715,4 \mathrm{Kcal}$. Ce mélange est naturellement riche en protéine, en minéraux et apporte l'énergie en quantité nécessaire pour contribuer à la couverture des

\section{MATÉRIELS ET MÉTHODES}

Matériels : Le matériel utilisé pour cette étude était une fiche de collecte d'informations sur l'identification, les mesures anthropométriques, renseignant également sur l'état physiologique et les traitements reçus à l'admission, ainsi que le suivi hebdomadaire de l'état nutritionnel des enfants. Elle a été établie et pré-testée au niveau du centre de santé intégré de Tessaoua. Les critères d'admission d'un enfant dans le programme de récupération sont mentionnés au niveau de l'inclusion. Quant au critère de sortie, il est basé essentiellement sur le gain de poids qui doit être supérieur ou égal à $400 \mathrm{~g}$ et le PB supérieur ou égal à $125 \mathrm{~mm}$ pour les cas modérés.

Méthodes: II s'agit d'une étude prospective réalisée auprès des enfants âgés de 06 à 59 mois atteints de malnutrition aigüe légère ou modérée, fréquentant les centres de santé intégré (CSI) pour bénéficier d'une ration de prise en charge de la malnutrition aigüe modérée en ambulatoire.

Site de l'étude : L'étude a été menée au Niger dans la région de Maradi, dans le département de Madarounfa plus précisément au niveau du CSI de la commune rurale de Dan-Issa. Le choix de cette zone est justifié par une forte prévalence de malnutrition globale supérieure á la moyenne du département. Pour le seul mois d'août 2019, le CSI a enregistré 1107 cas de malnutrition globale dont, 480 cas de malnutrition aigüe modérée, 177 cas de malnutrition aigüe sévère sans complication et 450 cas de malnutrition sévère avec complication.

\section{Population cible de l'étude}

Critères d'inclusion : les enfant des deux sexes âgés entre 06 et 59 mois atteints de malnutrition aigüe modérée ou légère selon les standards de l'OMS de 2006, dont:

- $\quad$ pour la malnutrition modérée

- le rapport poids/taille (P/T) doit être supérieur ou égal à moins 3 et strictement inférieur à moins 2 Z-score ;

- le Périmètre brachial (PB) doit être supérieur ou égal à $115 \mathrm{~mm}$ et strictement inférieur à $125 \mathrm{~mm}$;

- et absence d'œdèmes bilatéraux besoins nutritionnels des enfants. Outre son intérêt nutritionnel évident, cette bouillie enrichie, présente aussi d'autres bienfaits pour le bénéficiaire. Une étude d'acceptabilité menée à Tessaoua a montré que les enfants de moins de cinq ans pouvaient en consommer régulièrement afin d'en optimiser la qualité nutritionnelle.

- Pour la malnutrition légère, le rapport poids/taille $(P / T)$ doit être supérieur ou égal à moins 2 et strictement inférieur ou égale à moins 1,5 Z-score.

Echantillonnage : Un échantillonnage a été effectué parmi les mères ayant un enfant âgé de 06 à 59 mois, qui se sont présentées au CSI au moment de l'enquête. Pour avoir le nombre requis, le recrutement a été réalisé sur une période de 42 jours, soit six semaines, durée moyenne de prise en charge de la malnutrition aigüe modérée selon le protocole national de prise charge de la malnutrition aigüe au Niger. Les enquêteurs se sont présentés du lundi au vendredi, au CSI pendant les activités de vaccination couplées avec le suivi de croissance et le dépistage de la malnutrition.

Période de collecte de données: La collecte des données s'est déroulée du 23 août au 04 octobre 2019, au CSI de Dan-Issa, seul centre de la commune où les soins sont fournis aux enfants.

Déroulement de la collecte de données: Trois groupes ont été établis pour une supplémentation alimentaire :

*groupe1 ( $N=20$ ) où les enfants atteints de malnutrition aigüe modérée ont reçu de la bouillie à base de la farine de mil, la farine des tourteaux d'arachide enrichie avec la poudre de Maringá

* groupe2 ( $N=12)$ où les enfants atteints de malnutrition aigüe légère ont reçu le même produit alimentaire que le premier groupe

* groupe3 $(\mathrm{N}=12)$ où les enfants atteints de malnutrition aigüe modérée ont reçu du CSB++.

$\mathrm{Au}$ début de chaque rencontre, les mères étaient informées du but du projet et de son caractère académique. Les mères étaient aussi assurées de la confidentialité des informations.

Conduite de la supplémentation alimentaire: Chaque jour, une quantité de $200 \mathrm{~g}$ de farine de mil enrichie ou du CSB++ est distribuée aux enfants atteints de malnutrition aigüe (à consommer matin, midi et soir) sans arrêter l'allaitement ou modifier les repas habituellement pris par les enfants. Le mode de préparation de farines recommandé est le suivant : le 
sachet de $200 \mathrm{~g}$ est délayé dans $800 \mathrm{ml}$ d'eau bouillie et cuite pendant 5 à 10 minutes. Afin d'éviter les risques de partage, on demande aux mères de faire deux préparations par jour, matin et soir soit $(2 \times 100$ $\mathrm{g} / \mathrm{J})$. Pour le groupe3, l'approvisionnement en aliment de supplément CSB++ est fourni chaque deux semaines par le CSI (la dotation est assurée par le Programme Alimentaire Mondiale) et pour les groupes 1 et 2, l'approvisionnement a été fait par le chercheur principal chaque semaine, cependant le contrôle des consommations était assuré quotidiennement par deux enquêteurs. Le suivi des consommations ainsi que toutes autres observations étaient consignés dans des fiches de suivi.

Aspects éthiques: Ce projet de recherche a été présenté au comité national d'éthique et de déontologie pour la recherche en santé du Ministère de la Santé Publique du Niger qui l'a approuvé (voici le numéro d'approbation 38/2018/CNERS). Cette étude entend évaluer l'efficacité de la bouillie faite de la farine de mil, la poudre de moringa et le tourteau d'arachide et du sucre chez les enfants âgés entre 06-59 mois atteints de malnutrition aigüe modérée ou légère dans la commune rurale de Dan-Issa en vue de formuler des recommandations de santé publique dans le but d'améliorer la situation nutritionnelle et sanitaire des enfants Nigériens. Les données collectées comportent des noms aussi bien pour l'enfant que la personne en charge de l'enfant dans le seul but de faciliter la recherche des perdus de vue et le suivi. Aucun nom ne sera connu du public, car les noms sont codés.

Traitement et Analyse des données : La saisie des fiches de collecte et l'analyse des données ont été faites avec le logiciel SPSS 20, Word et Excel 2007.

Méthodes d'évaluation: Avant l'introduction du supplément, les statuts nutritionnel et sanitaire des enfants ont été déterminés grâce à un examen clinique et des mesures anthropométriques. L'impact de la supplémentation est ensuite recherché au bout de chaque semaine. A chaque période d'évaluation, les paramètres ont été recherchés par les mêmes mesureurs, c'est-à-dire les mêmes agents de santé du CSI.
Examen clinique : II a été assuré par les agents de santé supervisés par le médecin chef du CSI selon une fiche d'observation clinique identique pour tous. Lorsque les enfants étaient malades, des traitements ont été prescrits.

\section{Anthropométrie}

Mesure du poids : les enfants ont été pesés nus avec une balance SALTER d'une capacité de $25 \mathrm{Kg}$ avec une précision de $100 \mathrm{~g}$ près. La balance était installée sur une surface dure et plane. Elle est calibrée chaque matin à l'aide d'un poids étalon de $5 \mathrm{Kg}$.

Mesure de la taille: la taille des enfants a été déterminée avec une toise en bois sur laquelle est fixé un ruban étalonné en $\mathrm{cm}$. Les précautions suivantes ont été prises :

- la toise était verticale et le socle bien horizontal

- les enfants de plus de deux ans étaient toisés debout, déchaussés, pieds joints et se tenaient droits de façon à ce que la toise touche les talons, les fesses, et la nuque. La tête était bien redressée.

- les enfants de moins de deux ans étaient toisés couchés, bien à plat sur la planche

Pied à plat ; les talons contre la planche et la tête était bien redressée.

Indices: l'indice poids-pour-taille a été calculé par rapport aux références de l'OMS exprimés en Z-score (Standards des références OMS, 2006).

Périmètre brachial (PB): toujours le mesurer sur le bras gauche avec un $\mathrm{mm}$ de précision. Au préalable, il faut rechercher les deux extrémités de l'avant-bras, c'est-à-dire l'épaule, l'os de l'omoplate et le coude. Alors, la mi-distance se retrouve soit par mesure en $\mathrm{mm}$ de la longueur (et calculer la moitie). On ne doit pas trop serré et pas trop lâche. Pour prendre la mesure, il faut lire à l'endroit indiqué par les flèches qui correspondent à $0 \mathrm{~cm}$.

Évaluer le degré d'œdème: Appuyez avec le pouce sur le dessus des 2 pieds en même temps et gardez la pression pendant environ 3 secondes ou comptez doucement 1001, 1002, 1003. S'il y a œdème, une empreinte reste pendant un certain temps sur le dessus du pied où on a appuyé. L'œdème doit être observé sur les 2 pieds pour que l'enfant soit identifié comme étant œdémateux 


\section{RÉSULTATS}

Tableau 1 : Profils anthropométriques, nutritionnels et pathologiques des enfants atteints de malnutrition aigüe modérée pris en charge avec la bouillie enrichie au moringa ( $\mathrm{N}=20)$

\begin{tabular}{|c|c|}
\hline Variables & Population des mères et des enfants \\
\hline Age (mois) ${ }^{\alpha}$ & $12,20 \pm 4,336$ \\
\hline \multicolumn{2}{|l|}{ Sexe $\beta$} \\
\hline Masculin & $9(45)$ \\
\hline Féminin & $11(55)$ \\
\hline Poids moyen à l'admission (kilogramme) ${ }^{\alpha}$ & $6,630 \pm 0,746$ \\
\hline Taille (centimètre) ${ }^{a}$ & $68,90 \pm 4,429$ \\
\hline PB ${ }^{1}$ moyen à l'admission (millimètre) ${ }^{\alpha}$ & $119 \pm 3,728$ \\
\hline$P / T^{2}$ à l'admission $\geq-3$ et $<-2$ z-score ${ }^{\beta}$ & $20(100)$ \\
\hline \multicolumn{2}{|l|}{ Signes de complication à l'admission $\beta$} \\
\hline Diarrhée & $9(45)$ \\
\hline Fièvre & $8(40)$ \\
\hline Infections respiratoires aigue & $8(40)$ \\
\hline Vomissements & $3(15)$ \\
\hline \multicolumn{2}{|l|}{ Traitement diététique à l'admission $\beta$} \\
\hline Bouillie 200g/j (mil+moringa+tourteau d'arachide+sucre) & $20(100)$ \\
\hline Etre à mesure de préparer seule la bouillie à la maison $\beta$ & $20(100)$ \\
\hline Education nutritionnelle et démonstrations culinaires ${ }^{\beta}$ & $20(100)$ \\
\hline Gain de poids après 7 jours de traitement (gramme/jour) ${ }^{\alpha}$ & $73,92 \pm 73,84$ \\
\hline PB moyen après 7 jours de traitement (millimètre) ${ }^{\alpha}$ & $121,90 \pm 6,691$ \\
\hline \multicolumn{2}{|l|}{ Signe de complication après 7 jours de traitement ${ }^{\beta}$} \\
\hline Diarrhée persistante & $2(10)$ \\
\hline Paludisme présumé & $2(10)$ \\
\hline Refus de boire et de manger & $2(10)$ \\
\hline Consommation journalière moyenne de la bouillie (millimètre) ${ }^{\alpha}$ & $200 \pm 75,00$ \\
\hline
\end{tabular}

Note : ${ }^{\alpha}$ les valeurs sont exprimées en moyenne et écart- type., ${ }^{\beta}$ les valeurs sont exprimées en effectif et pourcentage

${ }^{1}(\mathrm{~PB})$ : périmètre brachial, ${ }^{2}(\mathrm{P} / \mathrm{T})$ : indice poids pour taille

Caractéristiques de l'échantillon et état nutritionnel des enfants : L'âge des enfants variait de 7 à 26 mois, avec une moyenne de 12,20 mois $\pm 4,336$. La distribution des enfants selon le sexe est répartie comme suit, $55 \%$ des enfants sont de sexe féminin et $45 \%$ de sexe masculin. L'analyse des résultats a révélé que la moitié $(50 \%)$ a un âge compris entre 6 et 11 mois et $50 \%$ de 12 à 26 mois. Tous les enfants sont dépistés malnutris aigue modérés selon la courbe des références OMS 2006, avec l'indice poids pour taille $(\mathrm{P} / \mathrm{T} \geq-3$ et $<-2$ Z-score) et le périmètre brachial (PB $\geq 115$ et $<125 \mathrm{~mm}$ ). Le poids moyen à l'admission était de $6,630 \mathrm{~kg} \pm 0,746$. Le périmètre brachial moyen à l'admission était de119 mm $\pm 3,728$. L'état physiologique et le traitement reçu ont été déterminés à l'admission. En effet, on a constaté que 7 enfants sur 20 avaient la diarrhée ; 10 enfants souffraient de fièvre, 3 enfants vomissaient, 8 enfants souffraient des infections respiratoires aiguës (la toux, le rhume, la pneumopathie); 1seul enfant a des douleurs abdominales et pleure surtout la nuit, 1 enfant a la dysenterie et 1 souffrait de conjonctivite. Chaque enfant a reçu le traitement médical selon sa maladie, cependant, aucun médicament contre la diarrhée n'a été donné.

Ration alimentaire des enfants admis :Concernant le traitement diététique, chaque enfant a reçu un sachet de $200 \mathrm{~g}$ par jour de farine de mil enrichie avec la poudre de moringa, la farine de tourteau d'arachide et le sucre, sans tenir compte de certains paramètres comme le poids et l'âge de l'enfant. Le premier jour, les bouillies sont préparées et servies sur place par une infirmière sous la supervision du chercheur principal. Les jours suivants, la consommation du repas se fait à domicile, chez la mère. Toutes les mères $(100 \%)$ ont bénéficié des séances d'éducation nutritionnelle et des démonstrations culinaires. Les enquêteurs font régulièrement des visites à domicile pour voir les 
conditions de préparation et de consommation jusqu'à la sortie (6 semaines au maximum). Toutes les mères $(100 \%)$ ont affirmé être à mesure de préparer la bouillie à la maison sans aide d'une tierce personne. L'analyse du tableau 1 fait ressortir que la consommation journalière moyenne de la bouillie est de $200 \mathrm{ml} \pm 75,00$. La consommation individuelle de la ration se fait en 3 repas (matin, midi et soir). Ces bouillies sont consommées autant que la bouillie ordinaire qu'ils ont l'habitude de boire (90\%). L'acceptabilité et la tolérance parfaite de cette bouillie ont été notées chez $90 \%$ des enfants. Cependant, $10 \%$ de cas de refus de boire a été noté.

Etat physiologiques des enfants à l'admission : A l'admission, $90 \%$ des enfants ont présenté des complications médicales. Les principales complications dont souffraient les enfants sont la diarrhée (45\%), la fièvre $(40 \%)$, les infections respiratoires aigües $(40 \%)$ et les vomissements (15\%). En effet, l'état général des malnutris s'est avéré net après une semaine de régime dans $90 \%$ des cas. Cependant, on a observé la persistance de la diarrhée chez $10 \%$ des enfants et l'apparition des symptômes d'un paludisme présumé chez $10 \%$ des enfants.

Efficacité de la bouillie enrichie et son impact sur les poids des enfants: L'analyse des résultats a fait ressortir trois modes de sortie. L'atteinte du poids cible de $400 \mathrm{~g}$ donc la guérison et le $P B \geq 125 \mathrm{~mm}$, les références et la non-réponse au traitement. Globalement, sur les 20 enfants malnutris modérés admis pour la prise en charge, 18 ont atteint les critères de sortie et ont été correctement déchargés (ce qui constitue un taux de succès au traitement de $90 \%$ ). Un malnutris sur 20 a été référé au CRENI de Madarounfa soit (un taux de référence de 05\%) et un seul enfant malnutris sur 20 a été considéré comme non répondant au traitement, ce qui constitue un taux de non-réponse de $05 \%$. La durée moyenne de séjour était de 14 jours. L'analyse des résultats a aussi révélé que tous les enfants qui souffraient de diarrhée $(100 \%)$, ont connu une amélioration rapide et une régularisation du transit intestinal au bout d'une semaine chez $75 \%$ des enfants avec le seul traitement diététique et plus tard chez les autres en raison de pathologies associées.

Tableau 2: Profils anthropométriques, nutritionnels et pathologiques des enfants atteints de malnutrition aigüe légère pris en charge avec la bouillie enrichie au moringa $(\mathrm{N}=12)$

\begin{tabular}{|c|c|}
\hline Variables & Population des mères et des enfants \\
\hline Age en mois ${ }^{\alpha}$ & $28,92 \pm 15,163$ \\
\hline \multicolumn{2}{|l|}{$\operatorname{Sexe}^{\beta}$} \\
\hline Masculin & $4(33,33)$ \\
\hline Féminin & $8(66,67)$ \\
\hline Poids moyen à l'admission en kilogramme ${ }^{\alpha}$ & $9,0 \pm 1,8$ \\
\hline Taille en centimètre ${ }^{a}$ & $80 \pm 2,90$ \\
\hline $\mathrm{PB}^{1}$ moyen à l'admission en millimètre ${ }^{\alpha}$ & $123,92 \pm 3,728$ \\
\hline$P / T^{2}$ à l'admission $\geq-2$ et $\leq-1,5$ z-score ${ }^{\beta}$ & $12(100)$ \\
\hline \multicolumn{2}{|l|}{ Signes de complication à l'admission $\beta$} \\
\hline Diarrhée & $12(100)$ \\
\hline Traitement médical à l'admission & Aucun \\
\hline \multicolumn{2}{|l|}{ Traitement diététique à l'admission $\beta$} \\
\hline Bouillie mil $200 \mathrm{~g} / \mathrm{j}$ enrichie (moringa+tourteau d'arachide+sucre) & $12(100)$ \\
\hline Education nutritionnelle et démonstrations culinaires ${ }^{\beta}$ & $12(100)$ \\
\hline Etre en mesure de préparer la bouillie à la maison $\beta$ & $12(100)$ \\
\hline Gain de poids après 7 jours de traitement en (gramme/jour) ${ }^{\alpha}$ & $65,19 \pm 27,22$ \\
\hline PB moyen après 7 jours de traitement (en millimètre) ${ }^{\alpha}$ & $130 \pm 6,691$ \\
\hline \multicolumn{2}{|l|}{ Signe de complication après 7 jours de traitement ${ }^{\beta}$} \\
\hline Boutons dans la bouche & $1(8,33)$ \\
\hline Refus de boire la bouillie & $1(8,33)$ \\
\hline Consommation journalière moyenne de la bouillie (en millilitre) ${ }^{\alpha}$ & $295 \pm 95,00$ \\
\hline
\end{tabular}


Caractéristiques de l'échantillon et état nutritionnel des enfants : L'âge des enfants variait de 07 à 51 mois, avec une moyenne de 28,92 mois $\pm 15,163$. II y a au total 12 enfants atteints de malnutrition aigüe légère $(P / T \geq-2$ et $<-1,5$ Z-score) dont 4 garçons et 8 filles. Tous les enfants (100\%) avaient la diarrhée à l'admission et qu'aucun traitement contre la diarrhée n'a été donné. L'état de santé a été déterminé, ainsi que le traitement reçu à l'admission. Pour le traitement diététique, chaque enfant a reçu un sachet de $200 \mathrm{~g}$ de farine de mil enrichie avec la poudre de moringa, la farine de tourteau d'arachide et le sucre par jour pendant 7 premiers jours. Toutes les mères $(100 \%)$ ont bénéficié non seulement des séances de sensibilisation sur l'hygiène et les conséquences de la malnutrition sur la santé, la croissance, le développement et la survie de l'enfant, mais aussi des démonstrations culinaires. Toutes les mères (100\%) ont affirmé être en mesure de préparer seule la bouillie à la maison.

Efficacité de la bouillie enrichie et son impact sur les poids des enfants: L'analyse des résultats $d u$ tableau 2 a révélé que 9 enfants sur 12 soit $(75 \%)$ ont connu une amélioration rapide de leurs états nutritionnel et sanitaire dès la première semaine (les 7 premiers jours) de prise en charge. Le gain de poids variait de 400 à $1100 \mathrm{~g}$ par semaine avec une moyenne de $65,19 \mathrm{~g} \pm 27,22$ par jour. On a aussi constaté que tous les 9 enfants ont un PB qui est supérieur ou égale à $125 \mathrm{~mm}$, quoiqu'ici le $\mathrm{PB}$ ne soit pas un critère d'admission ou de sortie. Parmi les 9 enfants sortis, aucun ne présentait un signe de complication médicale à la fin de la 1ère semaine. La consommation journalière de la bouillie est de $295 \mathrm{ml} \pm 95,00$. Donc, sur les 12 enfants atteints de malnutrition aigüe légère recrutés dans le cadre de la prévention de la malnutrition, tous $(100 \%)$ ont atteint nos critères de sortie $(400 \mathrm{~g})$ et ont été correctement déchargés (ce qui constitue un taux de succès pour la prévention de la malnutrition de 100\%). La durée moyenne de séjour était de 9 jours. Sous le seul traitement diététique avec la farine de mil, tourteaux d'arachide et moringa, il a été constaté une amélioration rapide et une régularisation du transit intestinal au bout d'une semaine de régime chez $90 \%$ des enfants.

Tableau 3 : Profils anthropométriques, nutritionnels et pathologiques des enfants atteints de malnutrition aigüe modérée pris en charge avec le $\mathrm{CSB}++(\mathrm{N}=12)$

\begin{tabular}{|c|c|}
\hline Variables & Population des mères et des enfants \\
\hline Age en mois ${ }^{\alpha}$ & $16 \pm 5,560$ \\
\hline \multicolumn{2}{|l|}{ Sexe ${ }^{\beta}$} \\
\hline Masculin & $5(41,66)$ \\
\hline Féminin & $7(58,34)$ \\
\hline Poids moyen à l'admission kilogramme ${ }^{\alpha}$ & $6,650 \pm 0,68$ \\
\hline Taille centimètre ${ }^{\alpha}$ & $69,67 \pm 4,053$ \\
\hline $\mathrm{PB}^{2}$ moyen à l'admission millimètre ${ }^{\alpha}$ & $118,58 \pm 2,999$ \\
\hline$P / T^{3}$ à l'admission $\geq-3$ et $<-2$ z-score ${ }^{\beta}$ & $12(100)$ \\
\hline \multicolumn{2}{|l|}{ Signes de complication à l'admission $\beta$} \\
\hline Diarrhée & $3(25)$ \\
\hline Fièvre & $2(16,66)$ \\
\hline Infections respiratoires aigues & $1(8,33)$ \\
\hline Vomissement & $2(16,66)$ \\
\hline Traitement médical à l'admission & Aucun \\
\hline Education nutritionnelle et démonstrations culinaires ${ }^{\beta}$ & $0(00)$ \\
\hline \multicolumn{2}{|l|}{ Traitement diététique à l'admission $\beta$} \\
\hline $\mathrm{CSB}++200 \mathrm{~g} / \mathrm{j}$ & $12(100)$ \\
\hline Gain de poids après 7 jours de traitement gramme/jour a & $20,53 \pm 23,15$ \\
\hline PB moyen après 14 jours de traitement millimètre ${ }^{\alpha}$ & $123 \pm 10,436$ \\
\hline \multicolumn{2}{|l|}{ Signe de complication après 14 jours de traitement ${ }^{\beta}$} \\
\hline Boutons dans la bouche & $1(8,33)$ \\
\hline Refus de boire la bouillie & $2(16,66)$ \\
\hline
\end{tabular}

Note : a les valeurs sont exprimées en moyenne et écart- typ ; $\beta$ les valeurs sont exprimées en effectif et pourcentage

$1(\mathrm{~PB})$ : périmètre brachial, ${ }^{2}(\mathrm{P} / \mathrm{T})$ : indice poids pour taille 
Caractéristiques de l'échantillon et état nutritionnel des enfants : L'âge des enfants variait de 8 à 24 mois, avec une moyenne de 16 mois $\pm 5,560$. La distribution des enfants selon le sexe était de 5 garçons et 7 filles. Tous les enfants sont dépistés malnutris aigue modérés avec le rapport poids pour taille $(P / T \geq-3$ et $<-2$ Zscore) et le périmètre brachial ( $P B \geq 115$ et $<125 \mathrm{~mm}$ ). L'état physiologique à l'admission a été déterminé. Les principaux symptômes sont: la diarrhée, la fièvre, les vomissements, les infections respiratoires aiguës (IRA). Pour le traitement diététique chaque enfant a reçu $200 \mathrm{~g}$ de CSB++ par jour pendant deux semaines. La distribution de la ration sèche n'est pas accompagnée par l'éducation nutritionnelle et les démonstrations culinaires. Pour les enfants malnutris pris en charge avec le CSB++, le suivi se fait toutes les deux semaines.

Efficacité du CSB++ et son impact sur les poids des enfants : L'analyse des résultats du tableau 3 a montré que 3 enfants sur les 12 soit (25\%) ont vu leurs états nutritionnel et sanitaire s'améliorés après 15 jours de prise en charge. Le gain de poids variait de 400 à $1000 \mathrm{~g}$ pour les deux semaines avec une moyenne de $20,53 \mathrm{~g} \pm 23,15$ par jour. On a aussi constaté que 2 enfants sur les 3 ayant atteints le poids cible de $400 \mathrm{~g}$, ont un PB qui est supérieur ou égale à $125 \mathrm{~mm}$.

Tableau 4 : Autres bienfaits de la bouillie de mil, la poudre de moringa et la farine de tourteau d'arachide et le sucre chez les enfants âgés de 6 à 59 mois constatés par les mères d'enfants

\begin{tabular}{lc}
\hline Variables & Effectif (\%) ${ }^{1}$ \\
\hline Augmente la force et le dynamisme & $18(90)$ \\
Arrête la diarrhée chez les enfants & $15(75)$ \\
Elimine la constipation et la dysenterie & $3(15)$ \\
Favorise l'évacuation des selles & $4(20)$ \\
Améliore l'état général des enfants & $3(15)$ \\
Stimule l'appétit chez l'enfant & $15(75)$ \\
Augmente la production du lait maternel & $4(20)$ \\
\hline
\end{tabular}

$(\%)$ : pourcentage

On a constaté que $90 \%$ des mères ont cité un apport énergétique important comme bienfait (force,

\section{DISCUSSION}

Selon les résultats de l'étude du groupe1 (tableau1), 10 enfants sur 20, atteints de malnutrition aigüe modérée soit $(50 \%)$ ont connu une amélioration de leurs états nutritionnel et sanitaire dès la première semaine de prise en charge. Le gain de poids variait de 400 à $2100 \mathrm{~g}$ avec une moyenne de 73,92g $\pm 73,84$ par jour. Donc, 10 enfants avaient un gain de poids supérieur ou égale à $400 \mathrm{~g}$ (poids cible) à la fin de la première semaine. On a aussi constaté que tous les 10 enfants avaient un PB qui est supérieur ou égale à $125 \mathrm{~mm}$ et qu'aucun enfant ne présentait un signe de complication médicale à la fin de la 1 ère semaine. Globalement, sur les 20 enfants malnutris modérés du groupe 1 admis pour la prise en charge, 18 ont atteint nos critères de sortie et ont été correctement déchargés, ce qui constitue un taux de succès au traitement de $90 \%$. Un enfant sur 20 a été référé au CRENI soit (un taux de référence de $05 \%$ ) et un seul enfant sur 20 a été considéré comme non-répondant au traitement, ce qui constitue un taux de non-réponse de $05 \%$. La durée moyenne de séjour était de 14 jours pour ce groupe. dynamisme), suivi de $75 \%$ des mères qui ont affirmé que la bouillie a un effet anti-diarrhéique efficace.

Ces résultats corroborent avec ceux d'une étude réalisée à Dakar, où les auteurs ont aussi observé une amélioration rapide des états nutritionnels et sanitaire des enfants pris en charge avec un produit alimentaire (le Supplétal). Le gain pondéral moyen observé était de $67,33+4,4$ grammes par semaine (Sall et al., 1992). Aussi, les résultats de cette étude corroborent à ceux de Diadié dans son étude réalisée au centre de récupération nutritionnelle de Guidan Idder qui a observé un effet bénéfique des quatre recettes, faites des aliments locaux sur le gain pondéral des enfants, surtout au cours des deux premières semaines de prise en charge (Diadié et al., 2012). Ainsi, dans une étude réalisée au Sénégal, les auteurs ont montré que l'apport de Moringa oleifera dans les repas se manifeste par une augmentation des teneurs en fer et en protéique. Cependant, les auteurs suggèrent que pour faire de Moringa oleifera un moyen d'enrichissement d'aliment dans la lutte contre la malnutrition et l'anémie par carence en fer, il faut nécessairement améliorer la biodisponibilité de son fer 
(Ndong et al., 2007). En effet, plusieurs travaux réalisés au Niger et ailleurs ont montré l'intérêt nutritionnel de l'enrichissement de la bouillie local avec d'autres ingrédients locaux dans la prise en charge de la malnutrition. C'est ainsi que, Degbey dans son étude sur l'efficacité du régime alimentaire enrichi en spiruline réalisée en 2008 concernant 56 enfants, âgés de 6 à 24 mois, admis dans le service de pédiatrie A de l'Hôpital National de Niamey, dont 45 étaient atteints de marasme, 3 de kwashiorkor et 8 d'une forme mixte, recevant chacun $10 \mathrm{~g}$ de Spiruline en poudre répartis en 2 prises journalières mélangées à la bouillie de mil durant 2 semaines, a révélé une nette amélioration de leur état nutritionnel (Degbey, 2008). Une autre étude réalisée au Niger sur l'enrichissement de spiruline en supplément alimentaire à ajouter dans les aliments locaux, au niveau des Centres de Soins Intégrés et cases de santé en 2009, a révélé que sur les 124 enfants souffrant de malnutrition et d'anémie, il a été observé 84 cas $(67,74 \%)$ de guérison (Goll, 2010). Nombreux travaux portant sur les effets de la spiruline sur la malnutrition, les infections à $\mathrm{VIH}$, la carence en vitamine $A$, l'anémie et la fonction immune ont montré des résultats satisfaisants (Azabji et al., 2010 ; Halidou et al., 2008 ; Wang et al., 2008 ; Selmi et al., 2011). Par rapport au groupe 2 en lien avec la prévention de la malnutrition, nos résultats ont montré que $100 \%$ des enfants atteints de malnutrition aigüe légère auxquels on a donné de la bouillie de mil enrichie avec la poudre de moringa, le tourteau d'arachide et le sucre ont tous observé un amélioration de leur état nutritionnel. Ce fort taux de succès au traitement s'explique par le fait que ces enfants ne sont pas très malades, sont vigoureux et ont un appétit bon ou modéré. En effet, l'alimentation supervisée, l'application des mesures d'hygiène et l'intérêt que les mères ont accordé aux soins des enfants suite à la réalisation des séances d'éducation nutritionnelle et les démonstrations culinaires auraient contribué à l'amélioration de l'état sanitaire et nutritionnel des enfants. Plusieurs études ont fait ressortir l'importance des soins des mères dans l'amélioration du bien-être des enfants (Santé Québec, 2008; Beaudry, 2006). Selon certains travaux, l'utilisation des aliments locaux permet de prévenir la malnutrition et améliore l'état nutritionnel des enfants. C'est le cas de la farine Misola, utilisant des ingrédients locaux, produite par les associations féminines a aussi permis d'améliorer l'état nutritionnel de plusieurs milliers d'enfants au Niger, au Mali, au Sénégal et au Burkina Faso (Association PASS, 2009). L'analyse des résultats du groupe 3 ont révélé que, 3 enfants seulement sur 12 recevant le CSB++ ont atteint les poids cible de $400 \mathrm{~g}$ et 2 sur les 3 , ont un PB supérieur à $125 \mathrm{~mm}$ au cours de deux semaines de prise en charge. Cela pourrait s'expliquer par le fait que certaines mères d'enfants malnutris utilisent le CSB à d'autres fins et que d'autres ne comprennent pas le sens du programma de prise en charge. En effet, la distribution de la ration sèche seule ne constitue le traitement. Les conseils alimentaires et la création de liens avec d'autres interventions et programmes de support social et économique constituent, en principe, les principaux éléments d'une intervention nutritionnelle. Aussi, une des raisons qui explique la non-amélioration de l'état nutritionnel des enfants de ce groupe, c'est la rupture fréquente du CSB++. En effet, pendant la période de collecte des données, les chercheurs ont constaté la rupture du CSB++ qui a duré plus d'un mois. Les enfants malnutris étaient abandonnés à eux même durant plusieurs jours sans ration sèche. Cependant, il faut souligner que, dans certaines régions du pays, la distribution supervisée de l'aliment thérapeutique prêt à l'emplois et l'utilisation du CSB++ aux jeunes enfants a permis d'éviter la prévalence élevée de la malnutrition sévère, cela a été observé par le MSF-Niger (MSF, 2010). Les résultats de cette étude ont mis en évidence certains inconvénients de l'utilisation du CSB++ dans la prise en charge de la malnutrition modérée qui se résument pour l'essentiel à la rupture fréquente du CSB++ (allant jusqu'à 1 à 2 mois), certaines mères l'utilisent à d'autres fins (vendent pour acheter du niébé, riz, huile... pour le besoin de la famille), les mères ne prennent pas soins de leurs enfants, les maintiennent dans la malnutrition pour continuer à en bénéficier. Les résultats de l'étude ont fait ressortir d'autres avantages de la bouillie enrichie avec le moringa, le tourteau d'arachide et le sucre. En effet, mis à part l'apport énergétique et protéique important pour prévenir et prendre en charge les enfants atteints de la malnutrition modérés, elle a aussi l'avantage d'arrêter la diarrhée et stimuler l'appétit chez l'enfant. Plusieurs études ont montré que la diarrhée représente, au plan mondial, la seconde cause de mortalité chez les enfants de moins de moins de 5 ans. Dans les pays en développement, la diarrhée cause $19 \%$ des décès d'enfants de moins de 5 ans, sans évoquer son incidence sur la malnutrition, qui cause à son tour, $5 \%$ des décès pour la même tranche d'âge (OMS, 2006). La diarrhée est une cause majeure de malnutrition et les enfants malnutris ont un plus grand risque d'avoir des diarrhées (OMS, 2015). Certaines mères ont évoqué comme 
avantage de la bouillie enrichie au moringa et tourteau d'arachide, l'augmentation de la production du lait

\section{CONCLUSION}

L'utilisation de la farine de mil, tourteaux d'arachide enrichie au moringa dans la prise en charge de la malnutrition parait très intéressante. En effet ce produit bien accepté et bien toléré chez les enfants malnutris, donne des résultats remarquables tant au plan clinique qu'anthropométrique. Aux termes de la présentation des résultats, Les auteurs ont confirmé que cette farine peut être utilisée pour prévenir ou combattre à long terme la malnutrition chez les enfants de moins de cinq ans au Niger. Cependant, la bouillie seule ne constitue pas le traitement, mais l'intervention dans sa globalité c'est-à-dire que la consommation de la bouillie supervisée, doit être couplée avec des mesures

\section{REMERCIEMENTS}

Les auteurs de cet article remercient le Ministère de la Santé Publique (MSP) du Niger qui a donné l'autorisation de mener cette étude et l'Institut de Santé Publique (ISP) de Niamey pour son appui financier. Les

\section{RÉFÉRENCES BIBLIOGRAPHIQUES}

Association PASS : Misola lutte contre la malnutrition avec les ressources du pays, Burkina Faso, 2009, 6 P

Azabji Kenfack M, Edie Dikosso S, Loni G, Onana A, Sobngwi E, Gbaguidi E, Nguefack T, Von der Weid D, Njoya O, Ngogang J. Efficacy of Spirulina platensis as a nutritional supplement in malnourished HIV-infected adults: A randomised, single-blind study. 2010.

Beaudry, M., S. Chiasson et J. Lauzière. Biologie de l'allaitement: le sein, le lait, le geste. Québec, Presses de l'Université Laval du Québec, 2006, 614 P.

Degbey $\mathrm{H}$, Hamadou B, Oumarou H.: Evaluation de l'efficacité de la supplémentation en Spiruline du régime habituel des enfants atteints de malnutrition sévère. In Charpy et al. (ed.) International Symposium on Cyanobacteria for Health, Science and Development: 104-108, 2006.

Fréderic, S. et Maurin S. : La faim dans le monde : dites un chiffre. MSF, 2009.

Goll C. : Quand les nigériens adoptent la Spiruline. Magazine - Santé. Niger, 2010, 1 page.

Halidou Doudou M, Degbey H, Daouda H, Leveque A, Donnen $P$, Hennart $P$, Dramaix-Wilmet M. : maternel qui est également un autre atout pour prévenir la malnutrition chez les enfants.

sanitaires, d'hygiène, d'éducation nutritionnelle des mères, un suivi médical régulier et une bonne prise de conscience des mères sur les conséquences néfaste de la malnutrition sur la santé, la croissance, le développement et la survie de l'enfant. Donc, un appui au Ministère de la Santé Publique par les ONG intervenant dans le domaine, en partenariat avec le PAM et I'UNICEF, permettrait un renforcement de la prévention de la malnutrition et l'intégration de la prise en charge de la malnutrition aiguë modérée avec les aliments locaux testés scientifiquement, dans le paquet minimum d'activité des CSI.

auteurs remercient également les participants à cette étude ainsi que le projet Misola particulièrement l'unité de production de Tessaoua.

Supplémentation en spiruline dans le cadre de la réhabilitation nutritionnelle : revue systématique. Revue d'Épidémiologie et de Santé Publique 56: 425-431, 2008.

INS/ME/F. Rapport final " évaluation nationale de la situation nutritionnelle par la méthodologie smart » octobre / novembre 2018.Institut National de la Statistique, Ministère de l'Économie et des Finances, République du Niger, 2019, P.180

Médecin Sans Frontière (MSF) : Nous voulons voir moins d'enfants mourir de malnutrition. Publication 29. 2010, 15p.

Ministère de l'Économie et des Finances. Institut National de la Statistique. Site de l'Institut National de la Statistique du Ministère de l'Économie et des Finances. http://www.statniger.org (site consulté le 02 septembre 2019).

Ndong M., Wade S., Dossou N., Guiro A. T., Gning R. D. : Valeur nutritionnelle du Moringa oleifera, étude de la biodisponibilité du fer, effet de l'enrichissement de divers plats traditionnels sénégalais avec la poudre des feuilles. African Journal of Food Agriculture. Nutrition and Development. 7 (3). 1-17, 2007. 
OCHA. (2016) Les bulletins humanitaires d'OCHA sont disponibles sur http://www.unocha.org/niger | www.unocha.org I www.reliefweb.int

Organisation Mondiale de la Santé (OMS). Principes directeurs pour l'alimentation du nourrisson et du jeune enfant âgés de 6 à 24 mois qui ne sont pas allaités au sein. Organisation Mondiale de la Santé, Genève, 2006, 27. P

Organisation Mondiale de la Santé (OMS) : Principes directeurs pour l'alimentation complémentaire de l'enfant allaité au sein. Organisation Mondiale de la Santé, Genève, 2006, 36. P

Organisation Mondiale de la Santé (OMS) : Alimentation du jeune enfant au Niger. http://www.MDGF_ 2015 Enfant, sécurité alimentaire, nutrition. (Site consulté le 01/01/2018).

Organisation Mondiale de la Santé (OMS). Normes de croissance de l'enfant: World Health Organization/Nutrition for Health and Development (NHD)/ Sustainable Development and Healthy Environments. Organisation Mondiale de la Santé, Washington, D.C, 2006, 6.P

Organisation mondiale de la santé et UNICEF. Normes de croissance OMS et identification de la malnutrition aiguë sévère chez l'enfant: déclaration commune de l'Organisation mondiale de la santé et du Fonds des Nations Unies pour l'Enfance. Organisation Mondiale de la Santé, Washington, D.C, 2009, 11.P

OUMAROU DIADIE H. : Pratiques d'alimentation du nourrisson et du jeune enfant au Niger : Etat des lieux, analyses technologique et nutritionnelle des aliments de complément et propositions d'amélioration. Thèse de Doctorat de l'Université Domaine : Sciences agronomiques

Spécialité : Nutrition Humaine. Niger, 2013, 122 P

Oumarou Diadié H., B. Abdourahamane et B. Moussa. Acceptabilité et efficacité des aliments de complément locaux proposés par les ONGs au Niger. J. Appl. Biosci. 56: 4089- 4096, 2012

Sall, M.G., N. Kuakuvi, NG. Mbaye et M. Fall. Essai d'un aliment de sevrage commercial dans le traitement des malnutritions protéinoénergétiques graves en milieu hospitalier : à propos de 76 cas. Médecine d'Afrique noire, 39 (6), 1992.

Santé Québec. Politique de périnatalité 2008 - 2018 : un projet porteur de vie. Québec, Ministère de la Santé et des Services Sociaux. Gouvernement du Québec, 2008, 174 P.

Selmi C, Leung PS, Fischer L, German B, Yang CY, Kenny TP, Cysewski GR, Gershwin ME. : The effects of Spirulina on anemia and immune function in senior citizens. Cellular \& Molecular Immunology, advance online publication 31 January 2011.

Wang J, Wang Y, Wang ZX, Li L, Qin J, Lai WQ, Fu Y, Suter PM, Russell RM, Grusak MA, Tang GW, Yin SA. : Vitamin A equivalence of spirulina $\beta$ carotene in Chinese adults as assessed by using a stableisotope reference method. American Journal of Clinical Nutrition 87: 1730-1737, 2008. 